\title{
A low-variation on-resistance CMOS sampling switch for high-speed high-performance applications
}

\author{
MohammadReza Asgari ${ }^{1}$ and Omid Hashemipour ${ }^{2 a)}$ \\ ${ }^{1}$ Microelectronic Lab, Shahid Beheshti University, G. C. Tehran, Iran. \\ ${ }^{2}$ Department of Electrical and Computer Engineering, Shahid Beheshti University, \\ G. C. Tehran, Iran. \\ a)hashemipour@sbu.ac.ir
}

\begin{abstract}
This paper presents a high-linear bootstrapped sampling switch for high-speed applications. The proposed switch utilizes bootstrapping technique of both NMOS and PMOS types simultaneously. Thus, results in a low-variation low-value on-resistance switch. Using this idea, the proposed switch considers reliability constrains, and operates in full-swing input range. Furthermore, it is suitable for standard n-well CMOS technology. Simulation results show that proposed switch achieves on-resistance variation of less than $4 \%$ through fullrange input signal.
\end{abstract}

Keywords: constant on-resistance, reliability, bootstrapping technique, high-speed applications

Classification: Integrated circuits

\section{References}

[1] S. K. Gupta, M. A. Inerfield, and J. Wang, "A 1-GS/s 11-bit ADC With 55-dB SNDR, 250-mW Power Realized by a High Bandwidth Scalable Time-Interleaved Architecture," IEEE J. Solid-State Circuits, vol. 41, no. 12, pp. 2650-2657, Dec. 2006.

[2] B. Razavi, Principles of Data Conversion System Design, IEEE Press, Piscataway, NJ, 1995.

[3] A. M. Abo and P. R. Gray, "A 1.5-V, 10-bit, 14.3-MS/s CMOS pipeline analog-to-digital converter," IEEE J. Solid-State Circuits, vol. 34, no. 5, pp. 599-606, May 1999.

[4] C. J. Fayomi, M. Sawan, and G. W. Roberts, "Low-Voltage Analog Switch in Deep Submicron CMOS: Design Technique and Experimental Measurements," IEICE Trans. Fundamentals, vol. E89-A, no. 4, pp. 1076-1087, April 2006.

[5] S. R. Sonkusale and J. V. Spiegel, "A low distortion MOS sampling circuit," IEEE International Symp. Circuits and Systems, vol. 5, pp. V-585V-588, Aug. 2002. 
[6] L. Wang, J. Yin, J. Wu, and J. Y. Ren, "Dual-channel bootstrapped switch for high-speed high-resolution sampling," Electron. Lett., vol. 42, no. 22, pp. 1275-1276, Oct. 2006.

[7] O. A. Adeniran and A. Demosthenous, "Constant-resistance CMOS input sampling switch for GSM/WCDMA high dynamic range $\Sigma \Delta$ modulators," IEEE Trans. Circuits Syst. I, Reg. Papers, vol. 55, no. 11, pp. 3234-3245, Nov. 2008.

[8] A. Galhardo, J. Goes, and N. Paulino, "Design of improved rail-to-rail low-distortion and low-stress switches in advanced CMOS technologies," Analog Integrated Circuits and Signal Processing, vol. 64, no. 1, pp. 13-22, Aug. 2009.

\section{Introduction}

Employing a high performance front-end track-and-hold $(\mathrm{T} / \mathrm{H})$ circuit is essential to improve the dynamic performance of a high-speed analog-to-digital converter (ADC). In high-speed applications in which the $\mathrm{T} / \mathrm{H}$ circuit must work in higher speed, a great care must be taken in choosing and designing of the $\mathrm{T} / \mathrm{H}$ circuit. For instance, in a time-interleaved ADC that contains $M$ channels, each converter operates at $f_{S} / M$ while the front-end $\mathrm{T} / \mathrm{H}$ typically operates at the total sampling frequency, $f_{S}[1]$. In a simple structure, the $\mathrm{T} / \mathrm{H}$ circuit includes a sampling switch and a capacitor which form a low-pass filter in the track-mode. The variation of sampling switch on-resistance $\left(R_{\text {on }}\right)$ causes dependence of time-constant of $\mathrm{T} / \mathrm{H}$ circuit on input signal and thus, produces harmonic distortion. The $R_{\mathrm{on}}$ variation also introduces frequency dependant nonlinearity error in the track-mode; especially in high frequency input signal, this nonlinear variation causes input-dependent phase shift and therefore affects linearity. This distortion is called the track-mode distortion [2]. In conventional bootstrapped sampling switch [3], this nonlinear signal-dependant variation is mainly due to the body effect. As a straightforward approach, scaling up of the aspect ratio $(W / L)$ of bootstrapped switch can reduce the $R_{\text {on }}$ variation but at the expense of increasing junction capacitance that needs larger bootstrap capacitance to alleviate charge sharing between bootstrap capacitance and the sampling switch. Considering of more power consumption due to the larger sampling capacitance, this is not a suitable method in high sampling rates. A reliable sampling switch in standard n-well CMOS technology with $R_{\text {on }}$ variation of less than $5 \%$, which is appropriate for high performance high-speed applications is proposed. In next Section, a background of previous reported bootstrapped switches is presented. The proposed switch is explained in Section 3. Simulation results are then shown in Section 4. Finally, conclusions are given in Section 5.

\section{Background}

Bootstrapping approach can be realized by charging of a capacitor in the hold phase and then utilizing it as a floating battery during the sampling 
phase for charging the sampling switch such that its gate tracks its source with a nearly constant voltage $\left(\sim V_{\mathrm{DD}}\right)$. In order to avoid degrading the long-term performance of CMOS device, which probably occurs due to the gate-oxide breakdown, hot electron, and punch-through effects, the voltage between main terminals of device (i.e. $V_{\mathrm{GS}}, V_{\mathrm{GD}}$, and $V_{\mathrm{DS}}$ ) must be kept within the supply voltage of that technology [3]. Because of the nature of bootstrapping technique and also the need for a charge pump circuit, the gate voltage of main switch and voltage of some paths exceeds $V_{\mathrm{DD}}$. However, in the conventional bootstrapped circuit, this problem has been solved applying some modifications such as using a cascaded transistor.

Bootstrapping technique is not able to cancel non-linearity impact of $R_{\text {on }}$ variation due to the body effect. So, several compensation techniques have been proposed $[4,5,6,7,8]$. In an n-well CMOS process, the direct connection of bulk to source [4] or bootstrapping of bulk terminal [5] during the sampling phase can only be realized with PMOS devices. Nonetheless, because of difference in parameters of NMOS and PMOS device $\left(\mu_{n} \approx 4 \times \mu_{p}\right)$, given an $R_{\text {on }}$ value, PMOS device requires more dimensions compared to NMOS one. In high-speed applications, this may be problematic since increasing aspect ratio introduces more errors such as charge injection. On the other hand, to ensure a correct performance, the time-constant of network should be low enough. Another approach is bootstrapping both NMOS and PMOS switches $[6,7,8]$. The circuit presented in [6] suffers from device reliability and is not suitable for rail-to-rail applications. In Fig. 2 in [6], the $V_{\mathrm{SG}}$ of $M 9$ in sampling phase and $V_{\mathrm{SG}}$ of $M 17$ in hold phase exceed their allowable limit and reach their worst case $2 \times V_{\mathrm{DD}}$ when input voltage is $V_{\mathrm{DD}}$. Since the gate of $M 17$ does not track its source with a shifted voltage, it does not pass the rail-to-rail signal properly. The CMOS switch proposed in [7] limits the peak input voltage because of leakage currents and latch-up. Since the gate of PMOS bootstrapped switch experiences negative voltage during the sampling phase, leakage currents from source and drain terminals to the bulk of the NMOS transistor which have to pass this negative voltage, become critical. In [8], using a linearizing method applied to a CMOS switch results improvement of linearity of equivalent conductance. Although, compare to conventional one maximum absolute value voltage of the circuit is less, however, some switches suffer from excessive voltage between their terminals (e.g. $M_{2 \mathrm{n}}, M_{7 \mathrm{n}}$, $M_{2 \mathrm{p}}$, and $M_{7 \mathrm{p}}$ in Fig. 2 in [8]). In addition, because of lower overdrive voltage the switch indicates higher $R_{\mathrm{on}}$ compare to conventional one.

\section{The proposed bootstrapped switch}

Bootstrapping of both NMOS and PMOS switches has the advantage of decreasing the $R_{\text {on }}$ value as well as reducing the $R_{\text {on }}$ variation. However, as explained in Section 2 some limitations are forced. This work proposes a new structure for bootstrapping of a CMOS switch considering reliability and rail-to-rail operation. The principle of bootstrapping a CMOS switch is shown in Fig. 1(a) and (b). Here, switches $M_{\mathrm{N}}$ and $M_{\mathrm{P}}$ are the main 
sampling switches. $M_{\mathrm{P}}$ is body effect compensated in the first order by connecting its bulk to source in sampling phase. Two floating batteries can be implemented by switch-capacitor structures. The gates of N-type and P-type switches track the input voltage with the functions $V_{\text {in }}+V_{\mathrm{DD}}$ and $V_{\text {in }}-V_{\mathrm{DD}}$, respectively. As a result, the equivalent switch's $R_{\text {on }}$ is as follows:

$$
R_{\mathrm{on}-\mathrm{CMOS}}=\frac{1}{\mu_{n} C_{o x}\left(\frac{W}{L}\right)_{n}\left(V_{\mathrm{DD}}-V_{\mathrm{Thn}}\right)+\mu_{p} C_{o x}\left(\frac{W}{L}\right)_{p}\left(V_{\mathrm{DD}}-V_{\mathrm{Th} 0 \mathrm{p}}\right)}
$$

By increasing both N-type and P-type switch sizes, their $R_{\mathrm{on}}$ are reduced leading to less time-constant. On the other hand, this increase results in more voltage error due to the charge injection and clock feedthrough. So, there is a trade-off in choosing aspect ratios. Fig. 1 (c) shows the proposed CMOS bootstrapped switch. The N-type part of proposed switch can be originally found in [3]. Proper biasing of the P-type part is achieved using some useful nodes in the N-type part of the circuit, so that the proposed CMOS switch maintains reliability as well as proper operation in the rail-torail input range. The bootstrapped switch works with a single phase clock. In the hold phase $C k n$, gate of $M_{\mathrm{N}}$ connects to ground through $M_{\mathrm{N} 11}$. At the same time, $M_{\mathrm{P}}$ is turned off across $M_{\mathrm{P} 5}$. In this phase, $C_{\mathrm{BOOST} 1}$ and $C_{\mathrm{BOOST} 2}$ charge to $V_{\mathrm{DD}}$. Because the gate of $M_{\mathrm{P}}$ is driven by negative voltage

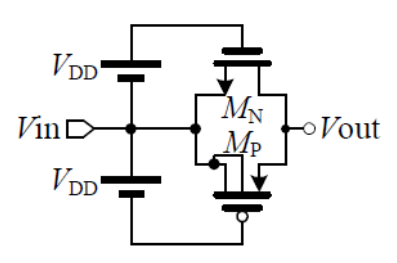

(a)

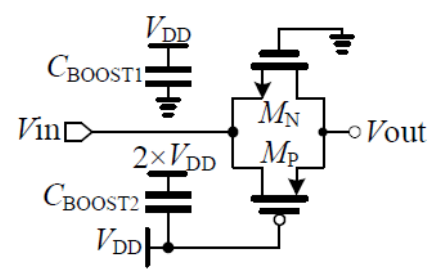

(b)

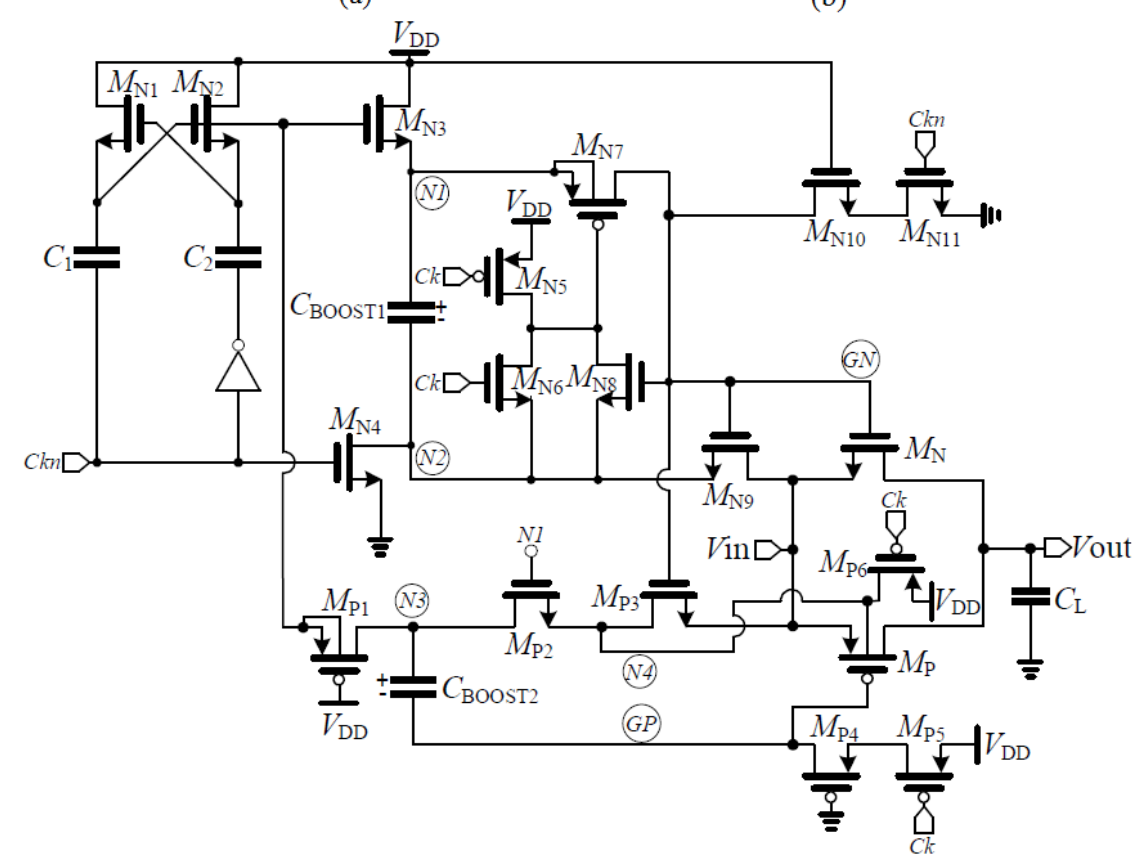

(c)

Fig. 1. CMOS bootstrapped switch (a) sampling phase (b) hold phase (c) proposed circuit. 
$\left(V_{\mathrm{in}}-V_{\mathrm{DD}}\right)$, any switch cannot be placed in this path. So, $C_{\mathrm{BOOST} 2}$ must be directly connected to $M_{\mathrm{P}}$. To provide the charge of $V_{\mathrm{DD}}$ on $C_{\mathrm{BOOST}}$, node $N 3$ reaches $2 \times V_{\mathrm{DD}}$ by $M_{\mathrm{P} 1} . C_{1}, C_{2}, M_{\mathrm{N} 1}$, and $M_{\mathrm{N} 2}$ form the conventional clock multiplier. $C_{\mathrm{BOOST} 1}$ is charged across $M_{\mathrm{N} 3}$ and $M_{\mathrm{N} 4} . M_{\mathrm{N} 7}$ and $M_{\mathrm{N} 9}$ isolate $M_{\mathrm{N}}$ from the boosting capacitor. During the sampling phase $C k, M_{\mathrm{N} 6}$ connects the gate terminal of $M_{\mathrm{N} 7}$ to the floating battery; consequently, $M_{\mathrm{N} 9}$, $M_{\mathrm{P} 3}, M_{\mathrm{P} 2}, M_{\mathrm{N}}$, and $M_{\mathrm{P}}$ turn on in a way that their $V_{\mathrm{GS}}$ values are fixed at $V_{\mathrm{DD}}$. Actually, the $V_{\mathrm{GS}}$ is less than $V_{\mathrm{DD}}$, because of parasitic capacitances at nodes $G N$ and $G P$. In the proposed switch, adding $M_{\mathrm{P} 2}$ plays two important roles: First, it avoids exceeding the $V_{\mathrm{DS}}$ of $M_{\mathrm{P} 3}$ from $V_{\mathrm{DD}}$. Second, $M_{\mathrm{P}}$ can be simply body effect compensated by connecting its bulk to the node $N 4$. In the mean time, properly operation of $M_{\mathrm{P} 2}$ and $M_{\mathrm{P} 3}$ are assured by connecting their gates to the nodes $N 1$ and $G N$, respectively. $M_{\mathrm{P} 3}$ also can be implemented as a PMOS device with its gate connected to node GP [4]. Node $N 4$ shorts the bulk of $M_{\mathrm{P}}$ to its source. Thus, the $V_{\mathrm{Th}}$ of transistor $M_{\mathrm{P}}$ becomes constant. In order to prevent punch-through, extra transistor $M_{\mathrm{N} 10}\left(M_{\mathrm{P} 4}\right)$ prevents the $V_{\mathrm{DS}}$ and $V_{\mathrm{GD}}$ of $M_{\mathrm{N} 11}\left(M_{\mathrm{P} 5}\right)$ exceed from $V_{\mathrm{DD}}$. Also, in order to avoid latch-up, bulk and source terminals of $M_{\mathrm{N} 7}$ and $M_{\mathrm{P} 1}$ are shorted. Note that at the beginning of sampling phase, $M_{\mathrm{N} 7}, M_{\mathrm{N} 9}$, and $C_{\mathrm{BOOST} 1}$ are responsible for rising time of $M_{\mathrm{N}}$. Similarly, $M_{\mathrm{P} 2}, M_{\mathrm{P} 3}$, and $C_{\mathrm{BOOST2}}$ direc tly affect the rise time of $M_{\mathrm{P}}$. Proper aspect ratio values will result in equal time-constants. Another issue is related to the fall time of $M_{\mathrm{N}}$ and $M_{\mathrm{P}}$. Any delay between turning off time of main switches leads to error in sampling capacitor and causes distortion. Consequently, a proper sizing of $M_{\mathrm{N} 10}, M_{\mathrm{N} 11}, M_{\mathrm{P} 4}$, and $M_{\mathrm{P} 5}$ is necessary. The proposed switch considers reliability because none of $V_{\mathrm{GS}}, V_{\mathrm{GD}}$, or $V_{\mathrm{DS}}$ values of switches exceed $V_{\mathrm{DD}}$ even at presence of rail-to-rail input signal.

\section{Simulation results}

Both conventional [3] and proposed bootstrapped switch are simulated in a standard $0.18 \mu \mathrm{m}$ CMOS technology model using Hspice simulator. A correct sizing of aspect ratios results in desired linearity; this can be achieved when both N-type and P-type switches have equal $R_{\mathrm{on}}$ values at the input commonmode voltage. In this design, $(W / L)_{n}=5 / 0.18$ and $(W / L)_{p}=21 / 0.18$ for proposed switch and its equivalent value for conventional one $(W / L)_{n}=$ 9.2/0.18 is chosen. Fig. 2 (a) shows $R_{\text {on }}$ value versus input signal amplitude. As can be seen, the proposed switch shows less $R_{\text {on }}$ variation. This variation is only $\sim 3.5 \%$ in proposed switch, whereas in conventional one is $24 \%$. Dynamic performance comparison is achieved using a differential $\mathrm{T} / \mathrm{H}$ circuit that includes two sampling switches and loading capacitors of $1 \mathrm{pF}$. A $1 V_{\mathrm{pp}}$ sinusoidal signal, $500 \mathrm{MHz}$ sampling clock, and $1.8 \mathrm{~V}$ supply voltage is applied to the circuits. SFDR plots of output versus input frequency from low to nyquist frequencies is shown in Fig. 2 (b). In high frequencies proposed switch shows more improvement where in near of nyquist frequency we have $13 \mathrm{~dB}$ improvement. Fig. 2 (c) presents SFDR results at different signal amplitudes 


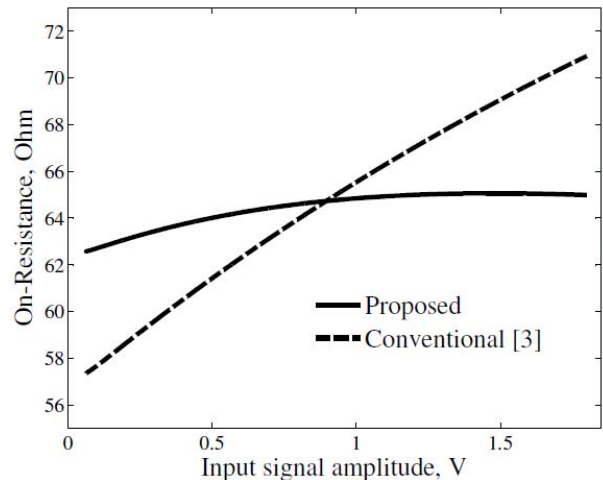

(a)

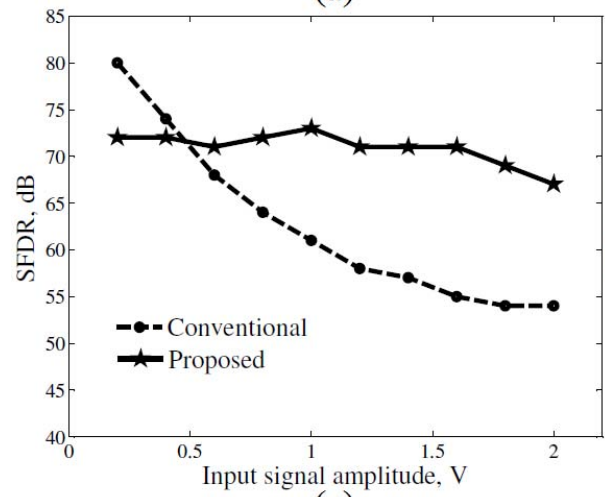

(c)

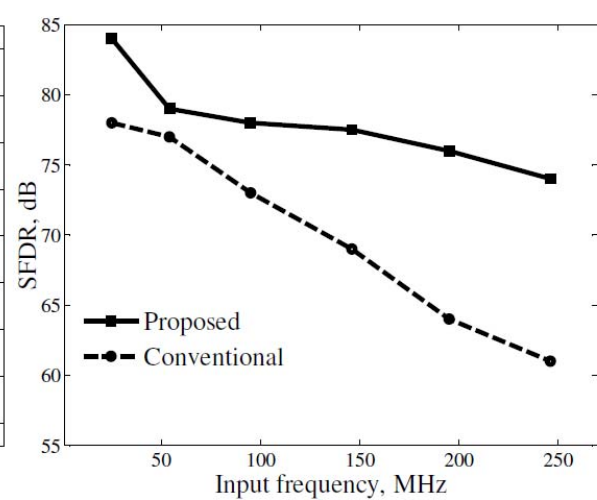

(b)

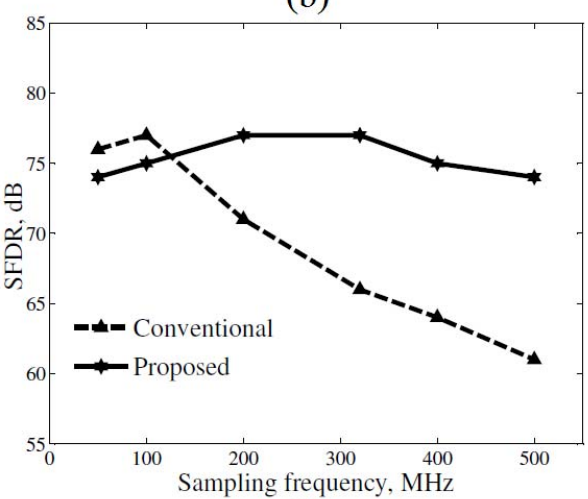

(d)

Fig. 2. Simulation results. (a) $R_{\mathrm{on}}$ variation against input signal amplitude (b) SFDR of fully-differential $\mathrm{T} / \mathrm{H}$ circuits versus input signal frequency (c) SFDR plots for $250 \mathrm{MHz}$ input frequency at different signal amplitudes (d) SFDR results versus different sampling frequencies with their equivalent nyquist input frequencies.

in $250 \mathrm{MHz}$ input frequency. The simulation result shows $15 \mathrm{~dB}$ SFDR improvement for $1.8 V_{\text {pp }}$ input signal in proposed switch versus convention one. In Fig. 2 (d), the SFDR plots are shown versus different sampling frequencies with their equivalent nyquist input frequencies. Simulation result shows that at different sampling frequencies the proposed switch indicates SFDR more than $73 \mathrm{~dB}$ whereas SFDR of conventional one decreases to $61 \mathrm{~dB}$ at $500 \mathrm{MHz}$ sampling rate. Table I confirms that remarkable improvement for reliability, rail-to-rail operation, and dynamic performance are achieved comparing to other CMOS switches. Note that some linearizing techniques such as bottom plate sampling and/or dummy switch is so effective in further dynamic

Table I. Comparison of CMOS bootstrapped switches.

\begin{tabular}{|l|l|l|l|}
\hline $\begin{array}{l}\text { CMOS boot- } \\
\text { strapped switches }\end{array}$ & $\begin{array}{l}\text { Max }\left|V_{\mathrm{GS}}\right|,\left|V_{\mathrm{GD}}\right|, \\
\text { or }\left|V_{\mathrm{DS}}\right|\end{array}$ & $\begin{array}{l}\text { Rail-to-rail } \\
\text { operation }\end{array}$ & $\begin{array}{l}\text { SFDR @ } 1 V_{\mathrm{pp}}, \\
246 \mathrm{MHz} \text { input }\end{array}$ \\
\hline$[6]$ & $2 \times V_{\mathrm{DD}}$ & No & $67 \mathrm{~dB}$ \\
\hline$[7]$ & $3 / 2 \times V_{\mathrm{DD}}$ & No & $57 \mathrm{~dB}$ \\
\hline$[8]$ & $V_{\mathrm{DD}}+V_{\mathrm{th}}$ & Yes & $71 \mathrm{~dB}$ \\
\hline Proposed & $V_{\mathrm{DD}}$ & Yes & $74 \mathrm{~dB}$ \\
\hline
\end{tabular}


performance improvement of switches especially at low frequency values.

\section{Conclusion}

Bootstrapping of both NMOS and PMOS switches have the advantage of both reducing the value of $R_{\mathrm{on}}$ and its variation. Using the above dual channel method a high-resolution reliable full-swing switch suitable for standard n-well CMOS technology is proposed. Simulation results indicate the effectiveness of proposed switch in high-speed applications.

\section{Acknowledgments}

The authors would like to thank the vice-presidency of Shahid Beheshti University for financial support of this work under grant $\sharp 600 / 1223$. 